The paper is presented at the conference "Complex analysis and its applications" (COMAN 2018), Gelendzhik - Krasnodar, Russia, June 2-9, 2018.

UDC 517.53

D. B. KATZ

\title{
BELTRAMI EQUATIONS REVISITED: MARCINKIEWICZ EXPONENTS AND PAINLEVE-TYPE THEOREM
}

\begin{abstract}
We deal with some new results on some types of Beltrami equations. There is a new approach involving the new metric characteristics: the Marcinkiewicz exponents. Another vision is applying the Cauchy-type integral representation to such equations. One more idea is to obtain analogs of some classical theorems for such equations.
\end{abstract}

Key words: fractals, non-rectifiable curves, boundary value problem, Riemann boundary value problem

2010 Mathematical Subject Classification: 30E25

1. Introduction. The Beltrami equation

$$
\bar{\partial} \phi=\mu \partial \phi, \quad|\mu(z)|<1,
$$

is one of the most natural and important generalizations of the CauchyRiemann equation (see [2]). Here, as usual,

$$
\bar{\partial}:=\frac{1}{2}\left(\frac{\partial}{\partial x}+i \frac{\partial}{\partial y}\right), \quad \partial:=\frac{1}{2}\left(\frac{\partial}{\partial x}-i \frac{\partial}{\partial y}\right)
$$

Theory of these equations has numerous applications in mechanics and physics [10]. Here we write about the jump problem for $\beta$-analytic functions, i.e. the problem of function $\phi(z)$ which vanishes in infinity, is continuously differentiable in $\mathbb{C} \backslash \Gamma$, satisfies the equation

$$
\bar{\partial} \phi(z)=\beta \frac{z}{\bar{z}} \partial \phi(z), \quad z \in \mathbb{C} \backslash \Gamma,
$$

(C) Petrozavodsk State University, 2018

\section{(cc) EY-NC}


and has continuous limit values $\phi^{ \pm}(t)$ in points $t \in \Gamma$ from domains $D^{ \pm}$ bounded by condition

$$
\phi^{+}(t)-\phi^{-}(t)=f(t), \quad t \in \Gamma,
$$

where $f$ is a given function. It is a particular case of the Riemann boundary value problem with $G \equiv 1$.

A lot of complex-variable boundary value problems use curvilinear integration over the boundary and such integrals as one of the basic methods of solution. For example, in the Riemann boundary value problem (see [4] and many others) a closed Jordan curve $\Gamma$ divides the complex plane to the finite domain $D^{+}$and the infinite one $D^{-}$and we seek for holomorphic in $\overline{\mathbb{C}} \backslash \Gamma$ functions that have boundary limits $\Phi^{ \pm}(t)$ from domains $D^{ \pm}$ correspondingly at any point $t \in \Gamma$, and these limits satisfy the condition

$$
\Phi^{+}(t)=G(t) \Phi^{-}(t)+g(t)
$$

In [4] the solutions of this problem are obtained in terms of the Cauchytype integrals

$$
\Phi(z)=\frac{1}{2 \pi i} \int_{\Gamma} \frac{v(t) d t}{t-z}, \quad z \notin \Gamma,
$$

and this is the classical result. However, such approach generates a new problem: the curvilinear integral $\int_{\Gamma} \cdot d z$ is defined only for rectifiable paths $\Gamma$, but the boundary value problem (2) itself can be set for any Jordan curve, including non-rectifiable (fractal and whatever) boundaries. This challenges us to extend the concept of curvilinear integration on nonrectifiable paths.

2. Integrations over non-rectifiable curves. We need some definitions first. As above, let $\Gamma$ be a closed Jordan curve of null plane measure on the complex plane $\mathbb{C}$ dividing it to domains $D^{+}$and $D^{-} \ni \infty$. If a function $\Upsilon(z)$ is continuously differentiable in $\mathbb{C} \backslash \Gamma$ and integrable together with its first partial derivatives near $\Gamma$ then we consider the mapping

$$
C_{0}^{\infty}(\mathbb{C}) \ni \omega \mapsto-\iint_{\mathbb{C}} \frac{\partial \Upsilon \omega}{\partial \bar{z}} d z d \bar{z},
$$

which is clearly a distribution. 
If the curve $\Gamma$ is rectifiable, and function $\Upsilon(z)$ has limit values $\Upsilon^{+}(t)$ and $\Upsilon^{-}(t)$ at any point $t \in \Gamma$ from $D^{+}$and $D^{-}$correspondingly, then by means of Green's formula

$$
-\iint_{\mathbb{C}} \frac{\partial \Upsilon \omega}{\partial \bar{z}} d z d \bar{z}=\int_{\Gamma}\left(\Upsilon^{+}(t)-\Upsilon^{-}(t)\right) \omega(t) d t .
$$

This last equality allows us to extend the concept of curvilinear integral on non-rectifiable curves.

Definition 1. Let $\Upsilon(z)$ be continuously differentiable in $\mathbb{C} \backslash \Gamma$ and integrable together with its first partial derivatives in a neighborhood of $\Gamma$. Then we call distribution (4) an integration of $\Delta \Upsilon d z$ over $\Gamma$ and denote it $\int_{\Gamma} \omega \Delta \Upsilon d z$.

Analogously, the distribution

$$
C_{0}^{\infty}(\mathbb{C}) \ni \omega \mapsto \int_{\Gamma} \omega \Delta \Upsilon d \bar{z}:=\iint_{\mathbb{C}} \frac{\partial \Upsilon \omega}{\partial z} d z d \bar{z},
$$

is an integration of $\Delta \Upsilon d \bar{z}$ over $\Gamma$.

Let us note that we do not require existence of the boundary values $\Upsilon^{ \pm}(t)$ at every point of $\Gamma$.

It is clear that if functions $\Upsilon_{1}$ and $\Upsilon_{2}$ coincide in an arbitrary small neighborhood of $\Gamma$ then they determine the same integration. Analogously, if $\omega_{1}$ and $\omega_{2}$ coincide in an arbitrary small neighborhood of $\Gamma$, then $\int_{\Gamma} \omega_{1} \Delta \Upsilon d z=\int_{\Gamma} \omega_{2} \Delta \Upsilon d z$ and $\int_{\Gamma} \omega_{1} \Delta \Upsilon d \bar{z}=\int_{\Gamma} \omega_{2} \Delta \Upsilon d \bar{z}$.

Such definition of the integration immediately generates the problem of uniqueness which is discussed further.

We also need the definition of the Hausdorff dimension (see, for instance [3]). Let $A \subset \mathbb{C}$ be a bounded set. Its $\lambda$-dimensional Hausdorff $r$-content equals to

$$
\mathcal{H}_{r}^{\lambda}(A)=\inf \left\{\sum_{k=1}^{n} r_{k}^{\lambda}: A \subset \bigcup_{k=1}^{n} B\left(x_{k}, r_{k}\right), x_{k} \in A, 0<r_{k} \leqslant r\right\},
$$

the $\lambda$-dimensional Hausdorff measure is

$$
\mathcal{H}^{\lambda}(A)=\lim _{r \downarrow 0} \mathcal{H}_{r}^{\lambda}(A),
$$


and

$$
d m_{H}(A)=\inf \left\{\lambda \geqslant 0: \mathcal{H}^{\lambda}(A)=0\right\}
$$

is the Hausdorff dimension of $A$.

The Hausdorff dimension of any plane set does not exceed two. It equals one for any rectifiable curve. One can find Hausdorff dimensions of a number of fractal curves in Wikipedia.

In what follows we use the local version of the Hausdorff dimension. We call the function $\mathfrak{h}(t)$ the local Hausdorff dimension of $\Gamma$, if any point $t \in \Gamma$ has a neighborhood $\gamma \subset \Gamma$ such that $d m_{H} \gamma \leqslant \mathfrak{h}(t)$.

Furthermore, we introduce the local Hölder condition. We say that a function $f(t)$ satisfies the Hölder condition with exponent $\nu \in(0,1]$ on a set $A \subset \mathbb{C}$ and write $f \in H_{\nu}(A)$ if

$$
h_{\nu}(f ; A):=\sup \left\{\frac{\left|f\left(t_{1}\right)-f\left(t_{2}\right)\right|}{\left|t_{1}-t_{2}\right|^{\nu}}: t_{1,2} \in A, t_{1} \neq t_{2}\right\}<\infty .
$$

Let us fix a finite family $E=\left\{t_{1}, t_{2}, \ldots, t_{n}\right\}$ of points of $\Gamma$ and a function $\nu: \Gamma \backslash E \mapsto(0,1]$. We refer a function $U(z), z \in \mathbb{C} \backslash \Gamma$, to the class $\mathcal{H}_{\nu}(\Gamma \backslash E)$ if any point $t \in \Gamma \backslash E$ has a neighborhood $N=N(t)$ in $\mathbb{C}$ such that $N \cap E=\emptyset$, and $U \in H_{\nu(t)}\left(N \cap D^{ \pm}\right)$.

Now we are ready to formulate the uniqueness theorem for integrations.

Theorem 1. Let functions $\Upsilon_{1,2} \in \mathcal{H}_{\nu}(\Gamma \backslash E)$ satisfy assumptions of Definition 1. If

$$
\nu(t)>\mathfrak{h}(t)-1, \quad t \in \Gamma \backslash E,
$$

then the equality

$$
\Upsilon_{1}^{+}(t)-\Upsilon_{1}^{-}(t)=\Upsilon_{2}^{+}(t)-\Upsilon_{2}^{-}(t), \quad t \in \Gamma \backslash E,
$$

means identity of integrations

$$
\int_{\Gamma} \cdot \Delta \Upsilon_{1} d z=\int_{\Gamma} \cdot \Delta \Upsilon_{2} d z, \quad \int_{\Gamma} \cdot \Delta \Upsilon_{1} d \bar{z}=\int_{\Gamma} \cdot \Delta \Upsilon_{2} d \bar{z} .
$$

This theorem is proved for $E=\emptyset$ in paper [5]. Its proof for finite set of discontinuities $E$ is absolutely analogous.

3. Marcinkiewicz exponents and local conditions. Now let us define the Marcinkiewicz exponents. Let $\Gamma$ be a closed Jordan curve on the complex plane dividing it to two domains $D^{+}$and $D^{-}, 0 \in D^{+}, \infty \in D^{-}$. 
When $r>0, t \in \Gamma, p>0$ we use notation $B(t ; r):=\{z:|z-t|<r\}$, $B^{ \pm}(t ; r):=B(t ; r) \cap D^{ \pm}$,

$$
I_{p}^{ \pm}(t ; r)=\iint_{B^{ \pm}(t ; r)} \frac{d x d y}{\operatorname{dist}^{p}(x+i y, \Gamma)}
$$

The inner and outer Marcinkiewicz exponents of the curve $\Gamma$ in it's point $t$ is defined by

$$
\mathfrak{m}^{ \pm}(\Gamma ; t):=\sup \left\{p: \lim _{r \rightarrow 0} I_{p}^{ \pm}(t ; r)<\infty\right\}
$$

We call the value

$$
\mathfrak{m}^{*}(\Gamma ; t):=\max \left\{\mathfrak{m}^{+}(\Gamma ; t), \mathfrak{m}^{-}(\Gamma ; t)\right\}
$$

the Marcinkiewicz exponent of the curve $\Gamma$ in the point $t$. Here integrals $I_{p}^{ \pm}(t ; r)$ increase with growth of the radius, thus the condition $\lim _{r \rightarrow 0} I_{p}^{ \pm}(t ; r)<\infty$ is tantamount to condition $\exists r>0: I_{p}^{ \pm}(t ; r)<\infty$.

From [6] we know that those values are not less than $2-d m \Gamma$ and not greater than 1 , where $d m$ stands for the Minkowski dimension. In particular, the Marcinkiewicz exponents of a rectifiable curve are equal to 1. In all further text we assume that the curve $\Gamma$ has null flat measure. Then $d m \Gamma<2$ and $\mathfrak{m}^{ \pm}(\Gamma ; t)>0$ in any point of the curve.

The Marcinkiewicz exponents are bound to the local properties of the contour. To use this fact properly, let us introduce the local version of the Hölder condition. We assume that there is a real function $v(t)$ on $\Gamma$ such that $0<\nu \leqslant v(t) \leqslant 1, t \in \Gamma$. We say that a function $f(t)$ defined on $\Gamma$ belongs to the class $H_{v}^{l o c}(\Gamma)$ if for any point $t \in \Gamma$ we can find a radius $r=r(t)>0$ such that the restriction of $f$ to the set $\Gamma \cap B(t ; r)$ satisfies the Hölder condition with exponent $v(t)$.

As shown in [5] and some other papers, the Marcinkiewicz exponents are of great use in the solution of the Riemann boundary value problem. Let us see how they apply to the Beltrami equation.

4. Marcinkiewicz exponents approach. The following approach was initially presented in [8]. We shall prove one rather technical lemma first. Before we prove it, let us prove an additional lemma that will help us further. First of all, let us prove

Lemma. If $f \in H_{v}^{\mathrm{loc}}(\Gamma)$, and exponent $p \geqslant 1$ satisfies the condition

$$
p<\frac{\mathfrak{m}(\Gamma ; t)}{1-v(t)}, \quad t \in \Gamma
$$


then the problem (9) has a solution, partial derivatives of which are integrable in power $p$ in any finite part of the plane.

Proof. Fix the value $0<m(t)<\mathfrak{m}(\Gamma ; t)$. From the definition of Marcinkiewicz exponents and the class $H_{v}^{\text {loc }}(\Gamma)$ it follows that for each point $t \in \Gamma$ there exists such radius $r=r(t)>0$ that at one time at least one of two conditions $I_{m(t)}^{+}(t ; r(t))<\infty, I_{m(t)}^{-}(t ; r(t))<\infty$ and the condition $\left.f\right|_{\Gamma \cap B(t ; k r(t))} \in H_{v(t)}(\Gamma \cap B(t ; k r(t)))$ hold, where $k>1$ is a constant. Circles $B(t, r(t))$ form the cover of $\Gamma$, and we can choose a finite subcover from it. Let us denote it by $B_{j}=B\left(t_{j}, r\left(t_{j}\right)\right)$, circles $B_{j}^{\prime}=B\left(t_{j}, k r\left(t_{j}\right)\right)$, $j=1,2, \ldots, n$, also cover $\Gamma$. Let us build the partition of unity corresponding to the last cover. This partition consists of functions $s_{j} \in C_{0}^{\infty}$ such that supps $_{j} \subset \overline{B_{j}^{\prime}}$ and $\sum_{j=1}^{n} s_{j}(t)=1$ when $t \in \Gamma$.

Now let us define the function $f_{j}$ on the union of the set $\Gamma_{j}:=\Gamma \cap \overline{B_{j}^{\prime}}$ and the circumference $C_{j}$ that bounds the circle $B_{j}^{\prime}$; It is equal to $s_{j} f$ on $\Gamma_{j}$ and to zero on $C_{j}$. It is clear that $f_{j} \in H_{v}\left(t_{j}\right)\left(C_{j} \cup \Gamma_{j}\right)$, and we can apply the Whitney extension operator to this function (see [11]). Due to well-known properties of this operator we get the function $f_{j}^{w}(z)$ defined on the whole complex plane with the following properties:

- it equals $f_{j}$ on $C_{j} \cup \Gamma_{j}$;

- it satisfies the Hölder condition with exponent $v\left(t_{j}\right)$ on the whole complex plane;

- it has continuous partial derivatives in $\mathbb{C} \backslash \Gamma_{j}$

and

$$
\left|\nabla f_{j}^{w}(z)\right| \leqslant \frac{c}{d i s t^{1-v\left(t_{j}\right)}(z, \Gamma)},
$$

where $c$ is a positive constant.

Moreover, $f_{j}^{w}(z)$ outside $B_{j}^{\prime}$ is identical zero, i. e. this extension coincides with $s_{j} f$ not only on $\Gamma_{j}$, but on all the contour $\Gamma$, and $\sum_{j=1}^{n} f_{j}^{w}(t)=$ $f(t)$ when $t \in \Gamma$.

Let there be a point $t_{j}$ where the condition $I_{m\left(t_{j}\right)}^{+}\left(t_{j} ; r\left(t_{j}\right)\right)<\infty$ is satisfied. Define $\psi_{j}(z):=f_{j}^{w}(z) \chi^{+}(z)$, where $\chi^{+}(z)$ is the characteristic function of the domain $D^{+}$. It is evident that

$$
\psi_{j}^{+}(t)-\psi_{j}^{-}(t)=f(t) s_{j}(t), \quad t \in \Gamma,
$$


and $\nabla \psi_{j}$ is integrable in power $p=\frac{m\left(t_{j}\right)}{1-v\left(t_{j}\right)}$ in any finite part of the plane. If $I_{m\left(t_{j}\right)}^{-}(t ; r(t))<\infty$, then we put $\psi_{j}(z):=f_{j}^{w}(z)\left(\chi^{+}(z)-1\right)$, and this function has have the same properties.

Sum of the functions $\psi_{j}, \psi:=\sum_{j=1}^{n} \psi_{j}$ satisfies (9). As the value $m(t)$ can be chosen arbitrarily close to $\mathfrak{m}(\Gamma ; t)$, the lemma is proved.

As the Beltrami equation (1) is a particular case of the Riemann boundary value problem with $G \equiv 1$, the following theorem is valid:

Theorem 2. If $f \in H_{v}^{\mathrm{loc}}(\Gamma)$ and

$$
v(t)>1-\frac{1}{2} \mathfrak{m}(\Gamma ; t), \quad t \in \Gamma,
$$

then the jump problem for $\beta$-analytic functions can be solved.

Proof. We study the more general case

$$
\psi^{+}(t)-\psi^{-}(t)=f(t), \quad t \in \Gamma,
$$

The integral operator

$$
T^{\beta}: \varphi \mapsto T^{\beta} \varphi:=-\frac{1}{(1-\beta) \pi} \iint_{\mathbb{C}} \frac{\varphi(\zeta) d \xi d \eta}{\zeta-z\left|\frac{z}{\zeta}\right|^{\theta}},
$$

where

$$
\theta=\frac{2 \beta}{1-\beta}, \quad \zeta=\xi+i \eta
$$

can be found in [12] by A. B. Tungatarov. If the function $\varphi$ in continuous in $\mathbb{C} \backslash \Gamma$, it's support is compact, and this function is integrable in power $q>2$ in $\mathbb{C}$, then this operator has the following features:

1) function $T^{\beta} \varphi$ is continuous in $\overline{\mathbb{C}}$, vanishes at infinity and satisfies the condition

$$
\left|T^{\beta} \varphi(z)-T^{\beta} \varphi(\zeta)\right| \leqslant\left. c|z| z\right|^{\theta}-\left.\zeta|\zeta|^{\theta}\right|^{\frac{2}{p}-1},
$$

where $\frac{1+\beta}{q}+\frac{1-\beta}{p}<1$, and $c$ is a positive constant, independent from $z$ and $\zeta$ 
2) operator $T^{\beta}$ is the right inverse operator to the differential operator $\bar{\partial}^{\beta}:=\bar{\partial}-\beta \frac{z}{\bar{z}} \partial$, i. e. $\bar{\partial}^{\beta} T^{\beta} \varphi=\varphi$; when $\varphi \in L^{1}$ this equation should be understood in the sense of S. L. Sobolev, though in points of continuity of $\varphi$ it is right for usual derivatives, too.

Let us study the function

$$
\phi_{0}(z):=\psi(z)-T^{\beta} \bar{\partial}^{\beta} \psi(z)
$$

where $\psi$ is the solution built in the proof of the lemma above. Thus, $\phi_{0}$ is a solution of the jump problem for $\beta$-analytic functions if first partial derivatives are integrable in power more than two. Due to the lemma, it is so under assumption of the theorem.

The condition of the theorem above are in some cases less bounding than the mentioned above results of Abreu-Blaya R., Bory-Reyes J., and Pena-Pena D. The example of a closed curve where in at least one point

$$
\mathfrak{m}(\Gamma ; t)>2-d m \Gamma
$$

is built in paper [9].

From this result we can easily get

Corollary. If $f \in H_{\nu}(\Gamma)$ and

$$
\nu>1-\frac{1}{2} \mathfrak{m}(\Gamma ; t), \quad t \in \Gamma,
$$

then the jump problem for $\beta$-analytic functions can be solved.

There is also a theorem on uniqueness of the solution in paper [9].

5. Another approach. Analogs of the classical theorems. This approach was widely developed in and was further developed in [7]. Let us study the Beltrami equation

$$
\bar{\partial} \phi=\beta \frac{f}{\bar{f}} \frac{\bar{f}^{\prime}}{f^{\prime}} \partial \phi,
$$

In paper [7] it was shown that if a function $\psi$ is continuous in closure of a domain $\mathrm{D}$ and has there partial derivatives integrable in power greater than 2 , then it can be represented as

$$
\psi(z)=\frac{1}{2 \pi i(1-\beta)} \int_{\Gamma} \frac{\psi(t) f^{\prime}(t) d t}{f(t)-f(z)\left|\frac{f(z)}{f(t)}\right|^{2 \alpha}}
$$




$$
+\frac{\beta}{2 \pi i(1-\beta)} \int_{\Gamma} \frac{f(t) \psi(t) \overline{f^{\prime}(t) d t}}{\overline{f(t)}\left(f(t)-f(z)\left|\frac{f(z)}{f(t)}\right|^{2 \alpha}\right)}
$$

We used the construction

$$
\phi(\zeta, z):=\frac{g(\zeta)}{g(\zeta)-g(z)}, g(z)=f(z)|f(z)|^{2 \alpha}
$$

to prove this fact in this paper.

The two analogs of the classical theorems (Liouville and Painleve) are valid.

Theorem 3. Let $f$ be a polynomial of degree $s, f(z)$ be analytic in $\mathbb{C}$ and function $\psi(z)$ satisfy the Beltrami equation (12) and the condition

$$
\liminf _{R \rightarrow \infty} R^{-k} \max \{|\psi(\zeta)|:|\zeta|=R\}=0 .
$$

in all complex plane. Then the function $\psi(z)$ can be represented as

$$
\psi(z)=P(g(z))
$$

where $P(z)$ is a polynomial of degree not greater than $m, m$ is the greatest of integers such that $m s(1+2 \alpha)<k$ and $g(z)=f(z)|f(z)|^{2 \alpha}$.

Proof. The theorem is proved immediately after we substitute the equation (13) into the analogous result from [1] instead of Tungatarov's formula.

One more analog of the classical theorems is the analog of the Painleve theorem. Painleve obtained the following result: if a function $F(z)$ is continuous in a domain $G$ and is holomorphic in $G \backslash \Gamma$, where $\Gamma$ is a rectifiable Jordan curve in $G$ then this function is holomorphic in $G$.

We have obtained the analog of the solution of the Beltrami equation (12) in our recent works. There is no restriction on $f$ to be holomorphic in all complex plane and we do not assume that $f$ is necessarily a polynomial. This result is presented and proved in [7].

Acknowledgment. This work was supported by RFBR grant 18-3100060 . 


\section{References}

[1] Abreu-Blaya R., Bory-Reyes J., Pena-Pena D., Vilaire J.-M. Riemann boundary value problem for $\beta$-analytic functions. International Journal of Pure and Applied Mathematics, 2008, vol. 42, no. 1, pp. $19-37$.

[2] Vekua I. N. Generalized Analytic Functions Nauka, 1988 (in Russian).

[3] Falconer K. J. Fractal geometry Wiley and Sons, 3rd edition, 2014.

[4] Gakhov F. D. Boundary Value Problems Nauka, 1977 (in Russian).

[5] Kats B. A., Katz D. B. Marcinkiewicz Exponents and Integrals over Non-rectifiable Paths. Mathematical Methods in Applied Sciences, 2016, vol. 39(12), pp. 3402-3410.

[6] Katz D. B. Marcinkiewicz exponents and their application in boundaryvalue problems. Russian Mathematics, 2014, vol. 58, no. 3, pp . 57-59.

[7] Katz D. B., Kats B. A.Integral Representations for Solutions of Some Types of the Beltrami Equations. Russian Mathematics, 2018, vol. 62 , no. 3, pp. 18-22. DOI: https://doi.org/10.3103/S1066369X18030039

[8] Katz D. B. Marcinkiewicz Exponents and Jump Problem for Beltrami Equation. Russian Mathematics, 2017, vol. 61, no. 6, pp. 37-43.

[9] Katz D. B. New metric characteristics of non-rectifiable curves with applications, Siberian Mathematical Journal, 2016, vol. 57, pp. 364-372 (in Russian).

[10] Monahov V. N., Boundary-value problems with free boundaries for elliptic systems of equations Nauka, 1977 (in Russian).

[11] Stein E. M., Singular Integrals and Differential Properties of Functions. Princeton University Press, 1970.

[12] Tungatarov A. B., Properties of certain integral operator in classes of summable functions. Izvestiya AN Kazakh. SSR., Ser. phys.-matem. nauki, 1985, vol. 132 , no. 5, pp. 58-62 (in Russian).

Received May 30, 2018.

In revised form, September 13, 2018.

Accepted September 15, 2018.

Published online September 18, 2018.

Kazan Federal University

18 Kremlyovskaya str., Kazan 420008, Russia

E-mail: katzdavid89@gmail.com 